Case
Reports

Elias Kfoury, MD

Abdelkader Almanfi, MD. MRCP (UK)

Kathryn G. Dougherty, CRTT, CVT

Zvonimir Krajcer, MD
Key words: Aged, 80 and over; aortic aneurysm, abdominal/complications; blood vessel prosthesis implantation/instrumentation/methods; endoleak/ etiology; endovascular procedures/instrumentation/ methods; kidney diseases/ complications/diagnosis; renal artery/abnormalities; treatment outcome

From: Michael E. DeBakey Department of Surgery (Dr. Kfoury), Baylor College of Medicine; and Department of Cardiology (Drs. Almanfi and Krajcer, and Ms Dougherty), Texas Heart Institute and CHI St. Luke's-Baylor St. Luke's Medical Center; Houston, Texas 77030

Address for reprints: Zvonimir Krajcer, MD, 6624 Fannin St., Suite 2780, Houston, TX 77030

E-mail: zvonkomd@aol.com

(C) 2016 by the Texas Heart ${ }^{\circledR}$ Institute, Houston

\section{Endovascular Abdominal Aortic Aneurysm Repair}

\author{
by Means of the Chimney Technique in a Patient \\ with Crossed Fused Renal Ectopia
}

Crossed fused renal ectopia, a congenital anomaly in 1 of 7,000 individuals, presents a challenge during endovascular treatment of abdominal aortic aneurysm. Most treatment approaches in these patients have involved open surgical repair of the aneurysm or endovascular repair with coverage of the ectopic renal artery. We present what we think is the first case of endovascular abdominal aortic aneurysm repair with use of the chimney technique (parallel stent-grafting) to preserve an ectopic renal artery, in an 88-year-old man who was at high risk for open surgery. In addition to the patient's case, we discuss the relevant medical literature. (Tex Heart Inst J 2016;43(3):232-5)

$\mathbf{R}$ epair of abdominal aortic aneurysm (AAA) can be complicated by congenital renal anomalies. In open repair of AAA, crossed fused renal ectopia often complicates surgical exposure and renal artery (RA) reimplantation. We report our alternative endovascular treatment of an elderly patient who had AAA and crossed fused renal ectopia.

\section{Case Report}

An 88-year-old man with an expanding, asymptomatic infrarenal AAA was referred to our institution. A computed tomogram (CT) showed a 5-cm maximal diameter of the AAA (Fig. 1). The AAA had a 25-mm-long infrarenal neck that was $23 \mathrm{~mm}$ in diameter proximally and $25 \mathrm{~mm}$ distally. The patient had a crossed-over ectopic right kidney fused with the left kidney; the ectopic RA arose directly from the AAA and was $5.3 \mathrm{~mm}$ in diameter. The distal aortic neck was $25 \mathrm{~mm}$ in diameter, and the diameters of the common iliac arteries were $11 \mathrm{~mm}$ (left) and $9.7 \mathrm{~mm}$ (right).

The patient's medical history included hypertension, type 2 diabetes mellitus, obstructive sleep apnea, and hyperlipidemia. He had also undergone multiple operations, including a transurethral prostatectomy, an exploratory laparotomy for trauma, and ventral hernia repair with use of mesh. His renal function was normal (serum creatinine level, $0.95 \mathrm{mg} / \mathrm{dL}$; and estimated glomerular filtration rate, $75 \mathrm{~mL} / \mathrm{min}$ ). He was at high risk for surgery because of his comorbidities, hostile abdomen, and crossed fused ectopic kidney, so we recommended endovascular aneurysm repair (EVAR) of the AAA. We intended to preserve the ectopic RA by using the chimney technique, also called the parallel stent-grafting or snorkel technique.

After we attained percutaneous access to both common femoral arteries by means of ultrasonographic guidance, we inserted 6F sheaths bilaterally. We introduced a 5F pigtail catheter into the abdominal aorta. An abdominal aortogram of the infrarenal AAA showed the ectopic right RA originating from the AAA (Fig. 2). We decided to perform EVAR with the parallel endograft oriented caudally, because the distance would be shorter and the angle less acute than if the graft were directed proximally. We then pre-closed the femoral arteries with use of a $10 \mathrm{~F}$ Prostar ${ }^{\circledR} \mathrm{XL}$ vascular closure device (Abbott Vascular; Redwood City, Calif). We inserted one 20F GORE ${ }^{\oplus}$ DrySeal sheath (W.L. Gore \& Associates, Inc.; Flagstaff, Ariz) for subsequent placement of the main endograft device through the left femoral artery, and another for snorkel and limb extension through the right femoral artery.

We selectively cannulated the accessory RA with use of a 5F Renal Double Curve catheter (Cordis Corporation, a Johnson \& Johnson company; Miami Lakes, Fla) and a 0.018 -in Hi-Torque Steelcore Peripheral Guide Wire (Abbott Vascular). Selective 
angiography of the ectopic right kidney was then performed (Fig. 3). We inserted a 6F PINNACLE ${ }^{\circledR}$ DESTINA$\mathrm{TION}^{\circledR}$ guiding sheath (Terumo Medical Corporation; Somerset, NJ) through the sheath in the right femoral artery. Next, we advanced and deployed the main endograft device-a $28 \times 16 \times 166$-mm Endurant ${ }^{\circledR}$ II AAA Stent Graft System (Medtronic, Inc.; Minneapolis, Minn) — through the sheath in the left femoral artery. We cannulated the contralateral gate with use of a 0.035 -in super-stiff GLIDEWIRE ${ }^{\circledR}$ (Terumo Medical) and deployed a $6 \mathrm{~mm} \times 10$-cm Viabahn ${ }^{\circledR}$ stent-graft (W.L. Gore) in the ectopic right RA (Fig. 4). We then de-

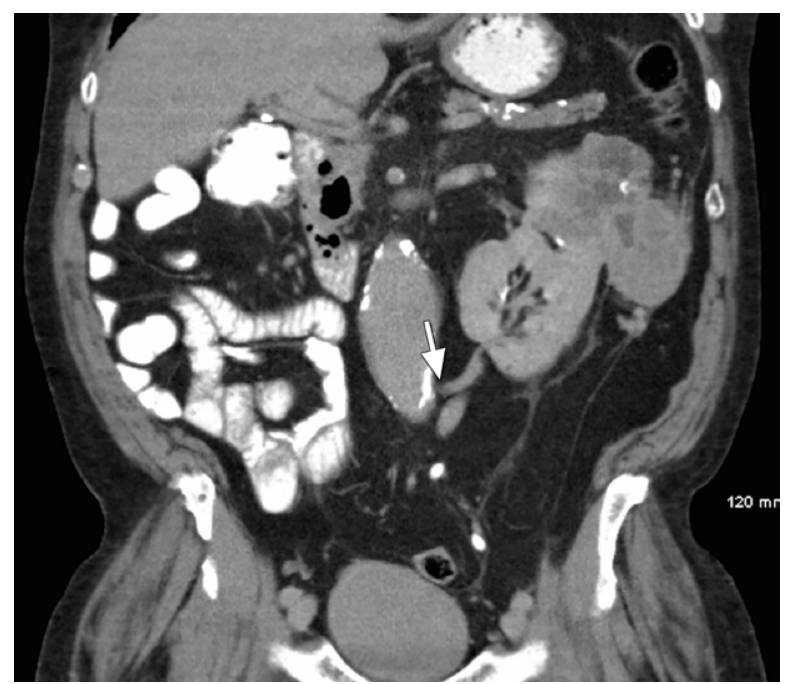

Fig. 1 Computed tomogram shows a 5-cm abdominal aortic aneurysm.

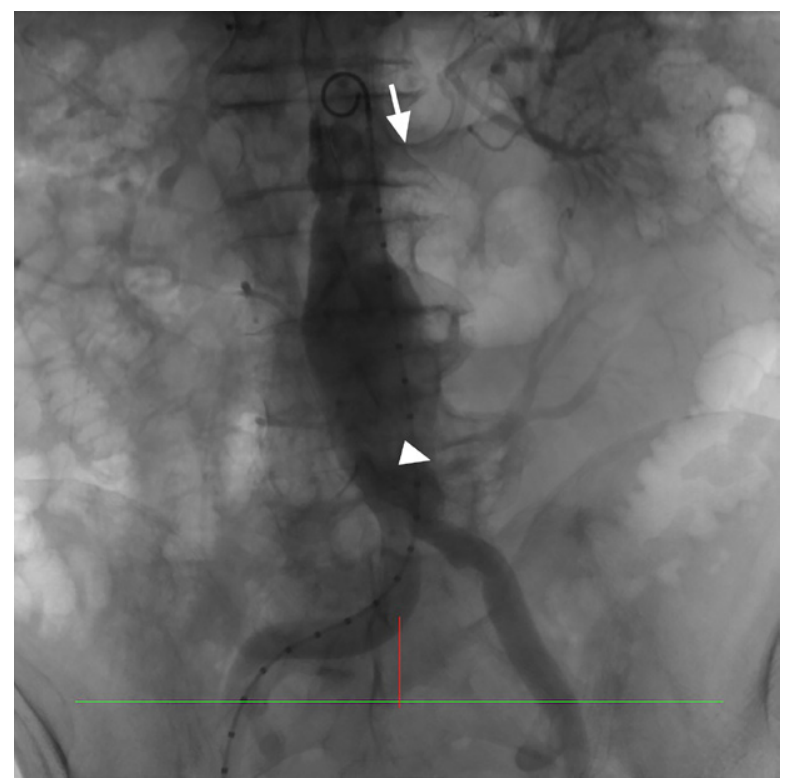

Fig. 2 Intraprocedural angiogram shows the origin of the left renal artery (arrow) and that of the ectopic renal artery from the aneurysm (arrowhead). ployed three $6 \times 29$-mm balloon-expandable PALMAZ ${ }^{\circledR}$ GENESIS $^{\circledR}$ stents (Cordis) inside the Viabahn stent, to prevent compression by the iliac extension limb of the endograft. Finally, we deployed a $16 \times 13 \times 93-\mathrm{mm}$ Endurant II iliac extension limb in the right common iliac artery. A completion angiogram showed adequate filling and no endoleak (Fig. 5).

The patient recovered uneventfully, and he was discharged from the hospital the next day. His serum creatinine level was $1.16 \mathrm{mg} / \mathrm{dL}$. A CT angiogram of his abdomen and pelvis one month later showed patent Endurant II and RA parallel endografts (Fig. 6). We

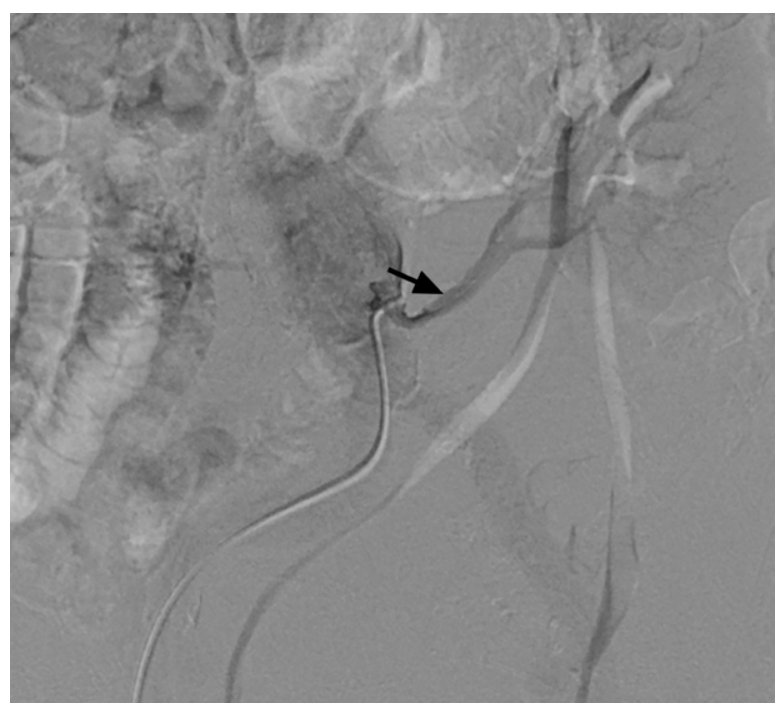

Fig. 3 Selective angiogram shows the origin of the ectopic renal artery (arrow).

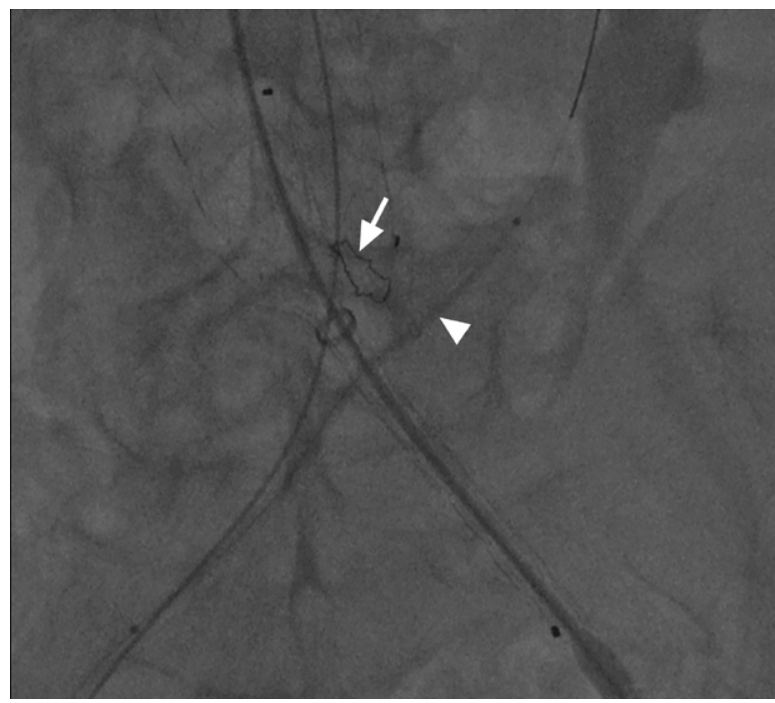

Fig. 4 Angiogram shows the Viabahn stent-graft in the ectopic renal artery (arrow) and the partially deployed Endurant II stent-graft (arrowhead) with the contralateral gate open and cannulated. 


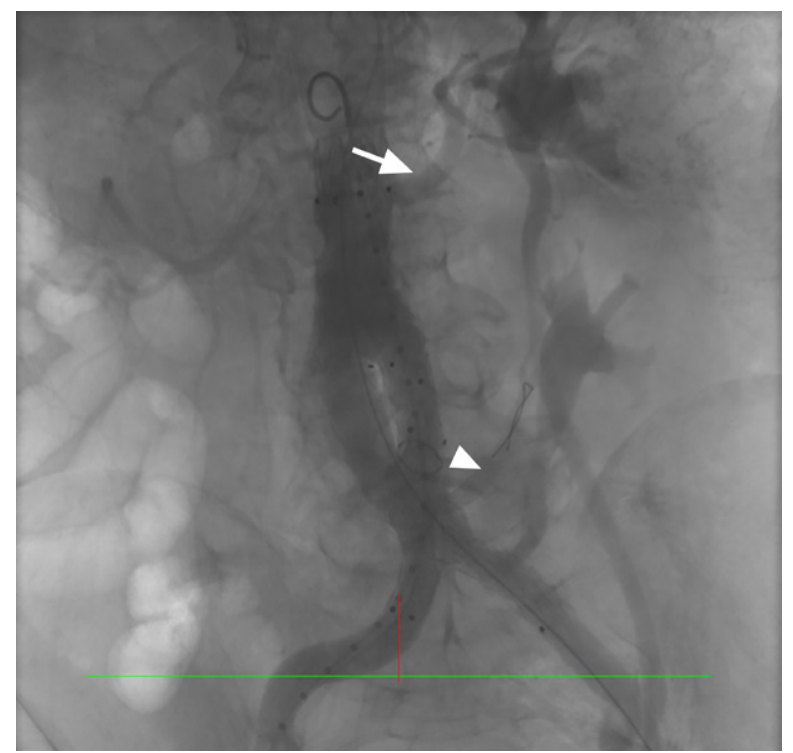

Fig. 5 Completion angiogram shows adequate filling of contrast medium into both the left renal artery (arrow) and the ectopic renal artery (arrowhead) without endoleak.

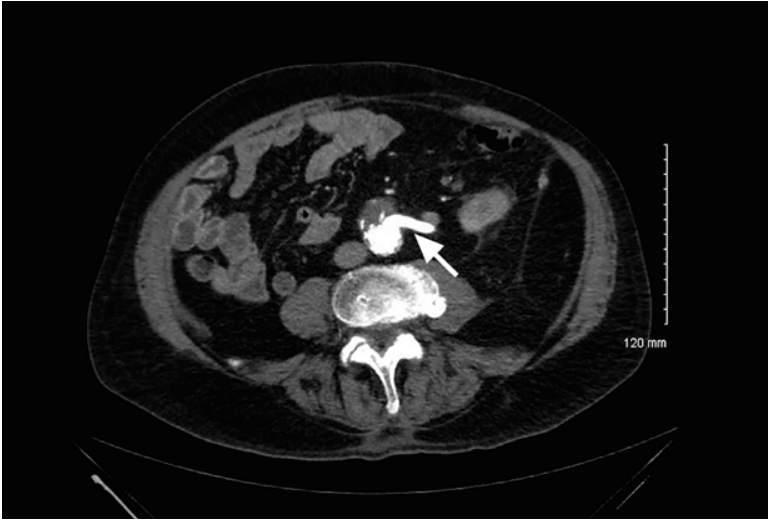

Fig. 6 Computed tomographic angiogram of the abdomen one month postprocedurally shows a patent stent-graft in the ectopic renal artery (arrow).

observed a type 2 endoleak originating from the inferior mesenteric artery, but no expansion of the aneurysmal sac. The patient was then monitored over time for possible embolization of the leaked blood.

\section{Discussion}

Open surgical repair of AAA is often challenging in patients who have renal anomalies, because of the frequently associated ureteral and renal vasculature anomalies. ${ }^{1}$ In autopsy studies, crossed fused renal ectopia was found in approximately 1 of 7,000 instances. ${ }^{1}$ This condition is thought to be secondary to abnormal migration of the ureteric buds. ${ }^{2,3}$ The most prevalent anatomic pattern is that in which the ectopic kidney crosses the midline and lies below the normal kidney: the ectopic renal ureter usually crosses the midline and inserts itself over the normal anatomic position. This increases the risk of injury during open surgery. ${ }^{3}$

We found only 3 reports in which crossover renal ectopia and AAA was treated by means of EVAR. In the first patient, EVAR was performed with "partial" coverage of the ectopic RA supply. ${ }^{4}$ In the 2 nd patient, a hybrid approach involved RA revascularization with open debranching surgery, followed by EVAR. ${ }^{5}$ In the 3rd patient, preoperative ectopic RA coiling was performed 25 days before EVAR. ${ }^{6}$ To our knowledge, ours is the first report of treating AAA and a crossed fused kidney by means of EVAR and the chimney technique to preserve the ectopic RA.

The parallel stent-graft technique was first described by Greenberg and colleagues ${ }^{7}$ for endovascular repair in patients who had challenging morphology of the AAA neck. The technique involves placing a stent into the RA to maintain perfusion and to enable more proximal coverage of the stent-graft. In published reports, covered stenting was typically used for the renal or visceral artery, and the chimney grafts were directed proximally. ${ }^{8}$ The technique has yielded good short- and midterm results; however, the long-term results still warrant evaluation. ${ }^{8}$

In our patient, we used a Viabahn stent-graft because of its flexibility in comparison with the more rigid balloon-expandable grafts, which might kink in the acute angle of the ectopic RA. However, no data support the idea that self-expanding stent-grafts are superior to balloon-expanding stent-grafts during use of the chimney technique. ${ }^{8}$

On the basis of our experience, we think that EVAR with chimney stenting of the ectopic RA is a feasible treatment for AAA and possibly safer than is open surgery or EVAR with coverage of the ectopic RAs, in patients whose renal anomalies involve ectopic RAs such as those encountered in crossed fused renal ectopia.

\section{References}

1. Crawford ES, Coselli JS, Safi HJ, Martin TD, Pool JL. The impact of renal fusion and ectopia on aortic surgery. J Vasc Surg 1988;8(4):375-83.

2. Bhatt K, Herts BR. Crossed fused renal ectopia. J Urol 2014; 191(2):475-6

3. McDonald JH, McClellan DS. Crossed renal ectopia. Am J Surg 1957;93(6):995-1002.

4. Barba Abad JF, Romero Vargas L, Tolosa Eizaguirre E, Rincon Mayans A, Rosell Costa D, Robles Garcia JE, Pascual Piedrola I. Association of crossed renal ectopia and aortic aneurism. Case report. Arch Esp Urol 2010;63(9):811-6.

5. Tadros RO, Malik RK, Ellozy SH, Marin ML, Faries PL, Vouyouka AG. A novel approach to the management of an inflammatory abdominal aortic aneurysm associated with crossed-fused renal ectopia. Ann Vasc Surg 2011;25(7):984. e9-14.

6. Schneider JR, Patel NH, Kim S, Verta MJ. Preemptive coil occlusion of major aberrant renal artery to allow endovascular 
repair of abdominal aortic aneurysm with crossed fused renal ectopia. Ann Vasc Surg 2014;28(5):1318.e1-6.

7. Greenberg RK, Clair D, Srivastava S, Bhandari G, Turc A, Hampton J, et al. Should patients with challenging anatomy be offered endovascular aneurysm repair? J Vasc Surg 2003;38 (5):990-6.

8. Moulakakis KG, Mylonas SN, Avgerinos E, Papapetrou A, Kakisis JD, Brountzos EN, Liapis CD. The chimney graft technique for preserving visceral vessels during endovascular treatment of aortic pathologies. J Vasc Surg 2012;55(5):1497503. 